\title{
Potential unfavorable impacts of BDNF Val66Met polymorphisms on metabolic risks in average population in a longevous area
}

Jun-Hua Peng ${ }^{1,2}$, Cheng-Wu Liu ${ }^{1,2}$, Shang-Ling Pan ${ }^{1,2^{*}}$, Hua-Yu Wu' ${ }^{1}$, Qing-Hua Liang ${ }^{3}$, Rui-Jing Gan ${ }^{1}$, Ling Huang ${ }^{1}$, Yi Ding ${ }^{1}$, Zhang-Ya Bian ${ }^{1}$, Hao Huang ${ }^{1}$, Ze-Ping LV ${ }^{3}$, Xiao-Ling Zhou ${ }^{1}$ and Rui-Xing Yin ${ }^{4}$

\begin{abstract}
Background: Brain-derived neurotrophic factor (BDNF) has been implicated in cognitive performance and the modulation of several metabolic parameters in some disease models, but its potential roles in successful aging remain unclear. We herein sought to define the putative correlation between BDNF Val66Met and several metabolic risk factors including BMI, blood pressure, fasting plasma glucose (FPG) and lipid levels in a long-lived population inhabiting Hongshui River Basin in Guangxi.

Methods: BDNF Val66Met was typed by ARMS-PCR for 487 Zhuang long-lived individuals (age $\geq 90$, long-lived group, $L G$ ), 593 of their offspring (age 60-77, offspring group, OG) and 582 ethnic-matched healthy controls (aged 60-75, control group, CG) from Hongshui River Basin. The correlations of genotypes with metabolic risks were then determined.

Results: As a result, no statistical difference was observed on the distribution of allelic and genotypic frequencies of BDNF Val66Met among the three groups (all $P>0.05$ ) except that AA genotype was dramatically higher in females than in males of CG. The HDL-C level of A allele (GA/AA genotype) carriers was profoundly lower than was non-A (GG genotype) carriers in the total population and the CG ( $P=0.009$ and 0.006 , respectively), which maintained in females, hyperglycemic and normolipidemic subgroup of CG after stratification by gender, BMI, glucose and lipid status. Furthermore, allele A carriers, with a higher systolic blood pressure, exhibited 1.63 folds higher risk than non-A carriers to be overweight in CG $(O R=1.63,95 \%$ $C$ I: $1.05-2.55, P=0.012$ ). Multiple regression analysis displayed that the TC level of LG reversely associated with BDNF Val66Met genotype.

Conclusions: These data suggested that BDNF 66Met may play unfavorable roles in blood pressure and lipid profiles in the general population in Hongshui River area which might in part underscore their poorer survivorship versus the successful aging individuals and their offspring.
\end{abstract}

Keywords: BDNF gene, Polymorphism, Metabolic risks, Longevity, Correlation

\footnotetext{
* Correspondence: slpan@gxmu.edu.cn

${ }^{1}$ Department of Pathophysiology, Guangxi Medical University, 22

Shuangyong Road, Nanning 530021, Guangxi, China

${ }^{2}$ Guangxi Colleges and Universities Key Laboratory of Human Development

and Disease Research, 22 Shuangyong Road, Nanning 530021, Guangxi,

China

Full list of author information is available at the end of the article
} 


\section{Background}

Brain-derived neurotrophic factor (BDNF) belongs to the neurotrophin family and plays essential roles in multiple processes associated with neuronal proliferation, differentiation and survival $[1,2]$. It is mainly expressed in central nervous system (CNS) including the cortex, the hippocampus and limbic structures and is linked to better cognitive status [3]. In addition, BDNF can cross the blood-brain barrier in both directions, accounting for more than $70 \%$ of serum BDNF in healthy humans $[4,5]$; serum BDNF concentration correlates closely with the cortical BDNF level and reflects the BDNF level in the brain [6].

Over the past decades, multiple lines of evidence have implicated that BDNF is not only stimulating for nerve growth and survival, but also exert nonneurotrophic effects over blood pressure, glucose, lipid and energy homeostasis in humans and model animals via central pathways involved in appetite regulation and energy expenditure [7-10]. It suppresses food intake, facilitates glucose uptake in the brain, reduces hepatic glucose production and converts white fat into brown fat in adipose tissue, leading to energy dissipation, lowered blood glucose and a lean phenotype; its downregulation in BDNF knockout mice and diabetic patients is associated with hyperphagic behavior, elevated blood glucose and cholesterol, and obese phenotype [10]. Together, these findings highlight the critical effects of BDNF on the maintenance of energy homeostasis and CNS functionality, perturbed BDNF signaling may confer metabolic risks which may further disrupt cognition and contribute to neurodegenerative pathologies such as Alzheimer's disease (AD), depression and Parkinson's disease (PD). It is thus reasonable to assume that long-lived individuals with better cognition might harbor beneficial BDNF genotypes and corresponding advantage profiles of metabolic parameters.

The human BDNF gene is located on the short arm of chromosome 11 at the boundary of $11 \mathrm{p} 13$ and $11 \mathrm{p} 14$ [11]. Several variants of BDNF gene have been reported and the most frequent is the functional Val66Met (c.196G > A, dbSNP: rs6265) polymorphism which substitutes a valine for a methionine at amino-acid residue 66 in the pro-BDNF protein, and the lower activity (Met) allele of this polymorphism is associated with reduced BDNF expression [12].

While BDNF Val66Met has been implicated as a genetic risk factor in several psychiatric disorders (e.g. schizophrenia, psychosis, major depression, anxiety, and eating disorders), cognitive compromised disorders (e.g. $\mathrm{AD}$ and $\mathrm{PD}$ ) and metabolic syndrome [13-15], very little data are available on its association with metabolic risks in general population, especially in long-lived individuals with good cognitive function.
The longevous population dwelling along the Basin of Hongshui River in Guangxi Province of China is a representative cohort of successful aging who have maintained better cognition [16] and escaped most of the common aged-related disorders including hypertension, diabetes, $\mathrm{AD}$ and $\mathrm{PD}$ and have been established as a cohort for longevity research for decades [17]. Herein, we evaluated the putative association between BDNF Val66Met polymorphism and metabolic risk factors in this population sample of long-lived men and women. This work was presented at The 26th Great Wall International Congress of Cardiology/Asia Pacific Heart Congress 2015/International Congress of Cardiovascular Prevention and Rehabilitation 2015 [18].

\section{Methods}

Study subjects

The participants investigated in the present work joined the ongoing Bama Longevity Genetic Study (BLGS) which was initiated in 2008 as described previously [18]. Herein, the long-lived group (LG) included 487 (men 200, women 287) nonagenerians/centenarians (mean age \pm SD, $93.28 \pm 2.87$ years) enrolled from native villages along Hongshui River Basin through Bama, Fengshan, Donglan and Du'an County of Guangxi Province, P. R. China. The offspring group (OG) comprised 593 natural children (son 333, daughter 260) of the studied nonagenerians/centenarians aged from 60 to 77 years (mean \pm $\mathrm{SD}, 67.55 \pm 8.02$ ). The control group (CG) consisted 582 (men 328, women 254) unrelated elderly aged from 60 to 75 years (mean $\pm S D, 65.27 \pm 9.32$ ) recruited from the same residential area as the oldest olds without familial history of longevity. All subjects were of ethnically Zhuang, apparently healthy with normal cognition and had no evidence of any chronic disorder. They were on typical Zhuang diet whose staple foods included maize, rice, bean products, sweet potato and various local vegetables. The meat they ate and the oil they used for cooking were mainly pork and lard, respectively. All subjects could do as much farm work or home work (e.g. baby caring) as they could. Participants with a history of myocardial infarction, stroke, hypertension, diabetes, AD and PD and a history of medication taking (at least 4 weeks prior to our enrollment) were excluded. The current study was approved by the Ethics Committee of Guangxi Medical University. All participants could walk to the village clinics which were usually half a mile away from their houses where they were given verbal informed consents and their fingerprints (to express consent) were obtained after they received a full explanation of the study. Written informed consents were not obtained because of their low educational level. The consent procedure was also approved by the Ethics Committee of Guangxi Medical University. An incentive of about ten dollars was provided to each participant in the study [19]. 


\section{Epidemiological survey}

Information on socio-demography and lifestyles was collected with standardized questionnaires. Anthropometric parameters including blood pressure, body height, body weight, waist circumference and body mass index (BMI) were measured or calculated in all groups as described previously [20, 21]. Hypertension was defined as systolic blood pressure $(\mathrm{SBP})>140 \mathrm{mmHg}$ and/or diastolic blood pressure $(\mathrm{DBP})>90 \mathrm{mmHg}$, and/or previous usage of anti-hypertensive medications prior to the survey. Normal weight, overweight, and obesity were defined as $\mathrm{BMI}<24$, $24-28$, and $>28 \mathrm{~kg} / \mathrm{m}^{2}$, respectively $[20,21]$.

\section{Biochemical analyses}

An overnight fasting venous blood sample of $8 \mathrm{~mL}$ was collected for each subject, $4 \mathrm{~mL}$ of which was for serum separation and subsequent serological detections while the remaining was for DNA extraction. Fasting plasma glucose (FPG) was measured immediately by a blood glucose meter (Accu-Chek Active, Roche, Germany). Total cholesterol (TC), triglycerides (TG), low density lipoprotein cholesterol (LDL-C) and high density lipoprotein cholesterol (HDL-C) levels were assayed by enzymatic methods as previously described [20]. Individuals with $\mathrm{FPG}>7.1 \mathrm{mmol} / \mathrm{L}$ and TC $>5.17 \mathrm{mmol} / \mathrm{L}$ and $/$ or TG $>1.70 \mathrm{mmol} / \mathrm{L}$ were defined as hyperglycemia and dyslipidemia, respectively [21].

\section{Sample preparation and genotyping}

Isolation of genomic DNA of leukocytes was performed according to standard procedures. Genotyping of BDNF Val66Met polymorphism was conducted by amplification refractory mutation system-polymerase chain reaction (ARMS-PCR) as described by Sheikh et al. [22]. The key principle for this methodology is that oligonucleotides with a mismatched 3' residue do not function as a primer in the PCR due to the lack of 3' exonucleolytic activity of Taq DNA polymerase. In brief, two sets of PCR primers were used in the PCR where the first set (P1, forward, 5'-CCTACAGTTCCACCAGGTGAGAA GAGTG-3' and P2, reverse, 5'-TCATGGACATGTT TGCAGCATCTAGGTA-3') was expected to the 401 bp region containing the SNP of interest, while the second set (P3 and P4, 5'-CTGGTCCTCATCCAACAGCTCT TCTATaAC-3' and 5'-ATCATTGGCTGACACTTTCG AACcCA-3', in which lower case letter indicated mismatched nucleotide) was allele specific and accounted for the $G \rightarrow A$ substitution. PCR amplifications were carried out in a $20 \mu \mathrm{L}$ reaction volume containing $25 \mathrm{ng}$ of template DNA, primers (P1, P2, P3 and P4, $1.25 \mu \mathrm{mol} / \mathrm{L}$ for each) and $10 \mu \mathrm{L}$ of $2 \times$ Taq Master Mix (Beijing ComWin Biotech Co.,Ltd, China) and were run in a Biometra PCR system (TProfessional standard, Germany) with a predenaturation at $94{ }^{\circ} \mathrm{C}$ for $5 \mathrm{~min}$, followed by 30 cycles of $94{ }^{\circ} \mathrm{C}$ for $45 \mathrm{~s}, 62.5{ }^{\circ} \mathrm{C}$ for $60 \mathrm{~s}$ and $72{ }^{\circ} \mathrm{C}$ for
$60 \mathrm{~s}$ and a final extension at $72{ }^{\circ} \mathrm{C}$ for $5 \mathrm{~min}$. PCR products $(8 \mu \mathrm{L})$ were resolved on a $2 \%$ agarose gel for $30 \mathrm{~min}$ at $80 \mathrm{~V}$ and stained with Golden View I (Beijing Solorbio Co., Ltd, China). The 401 bp band represented the control amplicon, whereas the 253 and 201 bp bands represented the $\mathrm{G}$ and $\mathrm{A}$ allele-specific amplicons. For the genotyping reliability, thirty randomly selected DNA samples (ten for each genotype) were sequenced and the sequencing results were all in agreement with that of PCR genotyping. Laboratory technicians who performed genotyping were masked to clinical and serological data.

\section{Statistical analyses}

All data were analyzed using the SPSS 16.0 statistical software package (SPSS Inc, Chicago, IL). Levels of quantitative variables were presented as mean $\pm \mathrm{SD}$ except that TG levels were in medians (interquartile) due to skewed distribution. Allelic and genotypic frequencies were calculated directly. Hardy-Weinberg equilibrium was computed for the expected genotype distribution using the standard goodness-of-fit test. Difference in genotypic and allelic distribution between groups was estimated with chi-square test, and odds ratio (OR) and 95\% confidential interval (CI) were used to indicate relative risk. The statistical evaluation for the categorical variables between groups was assessed by one-way ANOVA test. The association of BDNF genotypes with blood pressure, BMI, FPG and serum lipid variables was evaluated using analysis of covariance (ANCOVA). Multiple logistic analyses with stepwise modelling were used to evaluate the association of blood pressure, BMI, FPG and serum lipid levels with genotypes $(G G=1$, $\mathrm{GA}=2, \mathrm{AA}=3$ ) and several environment factors. In all hypothetical tests, two-tailed values of $P<0.05$ were considered statistically significant.

\section{Results \\ Population characteristics and metabolic measures}

Table 1 summarizes the demographic, clinical, and biochemical characteristics of the three groups investigated. OG and CG did not differ in terms of age $(P>0.05)$. After adjustment for age and sex, DBP, TC, and LDL-C were found to be higher while FPG was lower in LG as compared with OG and/or CG. The blood levels of FPG, HDL-C and LDL-C of OG were significant higher than that of CG.

\section{Genetic distribution}

The distribution of the BDNF Val66Met alleles fit the Hardy-Weinberg equilibrium. Its minor allele frequency (MAF) in LG, OG and CG was $43.3 \%, 41.1 \%$ and 41.9 respectively. Overall, the distribution of genotypic and allelic frequency of this SNP did not differ across groups $(P>0.05)$ except that females represented more AA genotype than 
Table 1 Population characteristic and metabolic parameters of participants

\begin{tabular}{|c|c|c|c|c|c|}
\hline Parameter & LG $(n=487)$ & OG $(n=593)$ & CG $(n=582)$ & $F\left(X^{2}\right)$ & $P$ \\
\hline Age (years) & $93.28 \pm 2.87^{* \boldsymbol{\Lambda}}$ & $58.35 \pm 9.06$ & $58.18 \pm 9.77$ & 3239.680 & 0.000 \\
\hline Male/Female & $200 / 287$ & $333 / 260$ & $328 / 254$ & 31.813 & 0.000 \\
\hline$W C(\mathrm{~cm})$ & $76.23 \pm 9.14$ & $75.08 \pm 9.27$ & $75.53 \pm 9.41$ & 0.340 & 0.712 \\
\hline $\mathrm{BMI}\left(\mathrm{kg} / \mathrm{m}^{2}\right)$ & $20.21 \pm 3.63$ & $21.68 \pm 3.00$ & $21.69 \pm 3.24$ & 0.022 & 0.978 \\
\hline FPG (mmol/L) & $4.65 \pm 1.18^{\boldsymbol{\Lambda}}$ & $4.97 \pm 1.80^{*}$ & $4.73 \pm 1.52$ & 8.070 & 0.000 \\
\hline $\mathrm{SBP}(\mathrm{mm} \mathrm{Hg})$ & $165.83 \pm 26.15$ & $137.81 \pm 22.96$ & $136.02 \pm 23.56$ & 0.618 & 0.539 \\
\hline $\mathrm{DBP}(\mathrm{mm} \mathrm{Hg})$ & $89.21 \pm 13.17^{* \boldsymbol{\Lambda}}$ & $85.15 \pm 11.22$ & $84.68 \pm 11.31$ & 4.020 & 0.018 \\
\hline $\mathrm{PP}(\mathrm{mm} \mathrm{Hg})$ & $76.61 \pm 23.74$ & $52.66 \pm 19.81$ & $51.34 \pm 18.40$ & 2.484 & 0.084 \\
\hline TC $(\mathrm{mmol} / \mathrm{L})$ & $5.09 \pm 1.00^{*}$ & $5.31 \pm 1.13^{*}$ & $4.88 \pm 1.00$ & 28.690 & 0.000 \\
\hline $\mathrm{TG}(\mathrm{mmol} / \mathrm{L})$ & $0.97(0.45)$ & $1.00(0.89)$ & $0.95(0.66)$ & 2.150 & 0.117 \\
\hline $\mathrm{HDL}-\mathrm{C}(\mathrm{mmol} / \mathrm{L})$ & $1.61 \pm 0.53$ & $1.71 \pm 0.57^{*}$ & $1.62 \pm 0.50$ & 3.353 & 0.035 \\
\hline LDL-C (mmol/L) & $2.99 \pm 0.87^{*}$ & $2.99 \pm 1.04^{*}$ & $2.61 \pm 0.92$ & 27.325 & 0.000 \\
\hline
\end{tabular}

Abbreviations: LG long-lived group, OG offspring group, CG control group, WC waist circumference, BMI body mass index, FPG fasting plasma glucose, $S B P$ systolic blood pressure, $D B P$ diastolic blood pressure, $P P$ pulse pressure, $T C$ total cholesterol, $T G$ triglyceride (presented as median and interquartile range), $H D L-C$ high-density lipoprotein cholesterol, $L D L-C$ low-density lipoprotein cholesterol

$\Delta_{\text {vs OG, }} P<0.05 ;{ }^{*}$ vs CG, $P<0.05$

males $(P=0.004$, Fig. 1a) in control group under codominant but not dominant model, which persisted through sex stratification $(P>0.05$ for all, Fig. 1a, b and c).

\section{Genotypes and metabolic risk factors}

Overall, no difference was observed between the impact of BDNF rs6265 A genotype (GA/AA) and non-A genotype (GG) on waist circumference (WC), BMI, FPG, TG and blood pressure in combined population and the three groups under investigation (Fig. 2a, b, c, d, and e) except that HDL-C level of A genotype carriers was dramatically lower than non-A carriers in the combined population $(P=0.009)$ and in the controls $(P=0.006)$ (Fig. 2f). These trends did not change basically through sex stratification (Fig. 3a-j). When BMI, FPG and lipid status were taken into consideration, the SBP level of A genotype carriers was noticeably higher $(P=0.023)$ (Additional file 1: Figure S1, I) while HDL-C was lower $(P=0.003)$ (Additional file 1: Figure $\mathrm{S} 1, \mathrm{~J})$ than non-A carriers in the normal BMI subgroups of the controls; lowered FPG level in the euglycaemic subfraction of LG $(P=0.038)$ (Additional file 2: Figure S2, C), increased SBP level in the hyperglycemic subfraction of OG $(P=0.041)$ (Additional file 2: Figure S2, G) and lowered HDL-C level in the hyperglycemic subfraction of CG $(P=0.001)$ (Additional file 2: Figure S2, J) were found in individuals harboring A genotype versus non-A genotype; a reduced FPG concentration in LG normolipidemic subclass $(P=0.025)$ (Additional file 3: Figure S3, C), a reduced BMI and HDL-C $(P=0.014$ and $P=0.004$, respectively) (Additional file 3: Figure S3, B and $\mathrm{H})$ in OG normorlipidemic subclass and a raised BMI level $(P=0.008)$ (Additional file 3: Figure S3, B) while a compromised HDL-C level $(P=0.031)$ (Additional file 3:
Figure S3, J) in CG normorlipidemic subclass were detected when A genotype bearers were compared with non-A genotype bearers. Relative risk estimates showed that allele A carriers have 1.63 folds higher risk to be overweight in CG $(O R=1.63,95 \% C I: 1.05-2.55)$ while exhibit less risk to be hyperglycemic $(O R=1.039,95 \%$ $C I=0.619-1.742)$ and hyperlipidemic $(O R=1.198,95 \%$ $C I=0.847-1.694)$ in LG, OG and CG as compared with non-A carriers. Together, these data mainly demonstrate that the mutant Met allele of BDNF gene may have different impact on metabolic parameters in different population and these impacts might be influenced by different BMI, FPG and lipid status.

\section{Correlation analyses}

Multiple linear regression analyses revealed that BDNF Val66Met genotype was reversely correlated with TC level in LG. No correlation was observed between BDNF Val66Met genotype and other metabolic parameters in the three studied groups (Table 2).

\section{Discussion}

Instead of finding salutary genotypes of BDNF gene which may account in part for better preservation of health in the oldest olds and their offspring, we noted unexpectedly an overrepresentation of BDNF 66 Met and its adverse correlation with several metabolic parameters in the general population living in the same area as the long-lived families, which can also explain why local residents have higher morbidity and mortality as compared to individuals with exceptional longevity. These results are in concert with some but not all observations from other research groups. Here, the effect of this SNP on several common metabolic risks is discussed basing on our data. 


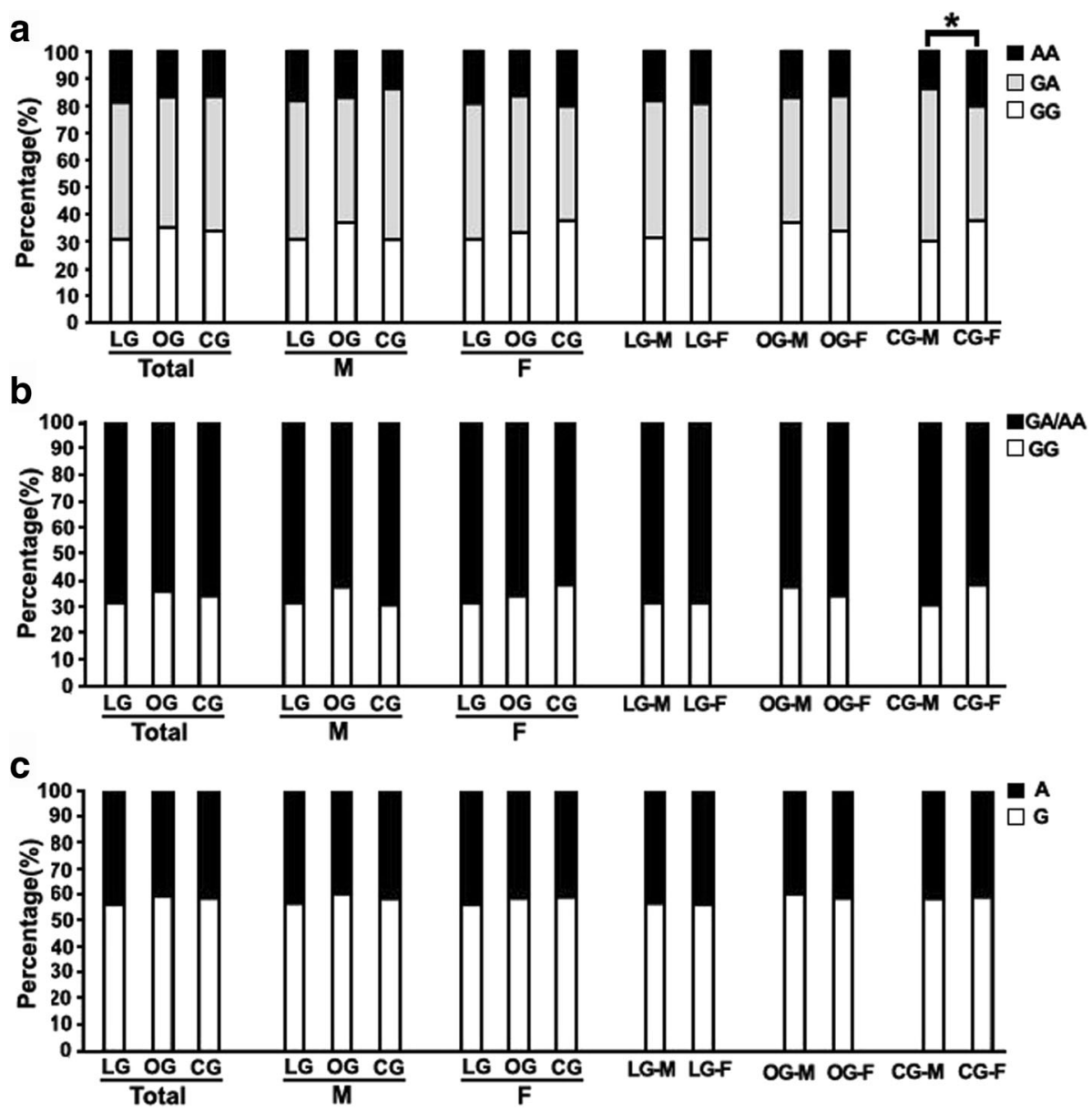

Fig. 1 Comparison of genotypic and allelic frequencies of the BDNF Val66Met among groups. a Genotypic frequency under co-dominant model, females in CG represented more AA genotype than males (Chi-square test, $\chi^{2}=11.263, P=0.004$ ). $\mathbf{b}$ Genotypic frequency under dominant model, no difference among groups and between sexes. c Allelic frequency, no difference among groups and between sexes. ${ }^{*} P<0.05$. Abbreviations: LG, long-lived group; OG, offspring group; CG, control group; M, males; F, females

\section{BDNF Val66Met and obesity}

Obesity has been so common and has emerged as a worldwide health problem due to its association with increased risk of metabolic-related disorders. BMI is one of the most important indicators for obesity assessment. Several previous studies had implicated that the derived Met allele of BDNF gene might down-regulate BDNF, raise blood glucose concentration and predispose individuals to obesity $[12,13,23,24]$. In the present work, BDNF Met allele carriers with normal BMI and lipid levels in OG displayed lower BMI whereas Met carriers with normal lipids in CG exhibited higher BMI level and risk of overweight, demonstrating a different impact pattern of BDNF Met on body shape between the offspring of nonagenarians and the age-matched controls. These findings are in accordance with those in several previous studies but not in others. For instance, Estonian adolescent girls bearing BDNF Val allele tended to have lower BMI than who bear Met allele; Croatian Caucasian children and adolescents who presented one or two Met alleles were prone to obesity; Puerto Rican women living in Boston with the GA or AA genotype were 50\% more likely to be overweight compared to GG carriers [25-27]. Mounting experimental evidence supports this association of Met variant with obesity: the derived Met allele reduces the expression of BDNF, which plays pivotal roles in the inhibition of excess calorie uptake (e.g. vigorous appetite, bad dietary behavior and sedentary lifestyle) and in the enhancement of energy expenditure (e.g. acceleration of brown fat metabolism and the degeneration of serum level of TG, TC, and free fatty acids and improvement of insulin resistance) $[23,28,29]$. On the contrary, Met allele was either linked to thin phenotype in British females, Chinese Han adolescents and Korean smokers [30-32] or lacked of relationship with obesity in German extremely obese children and adolescents and underweight students [33]. The inconsistency of these results indicates the uncertain association between BDNF 

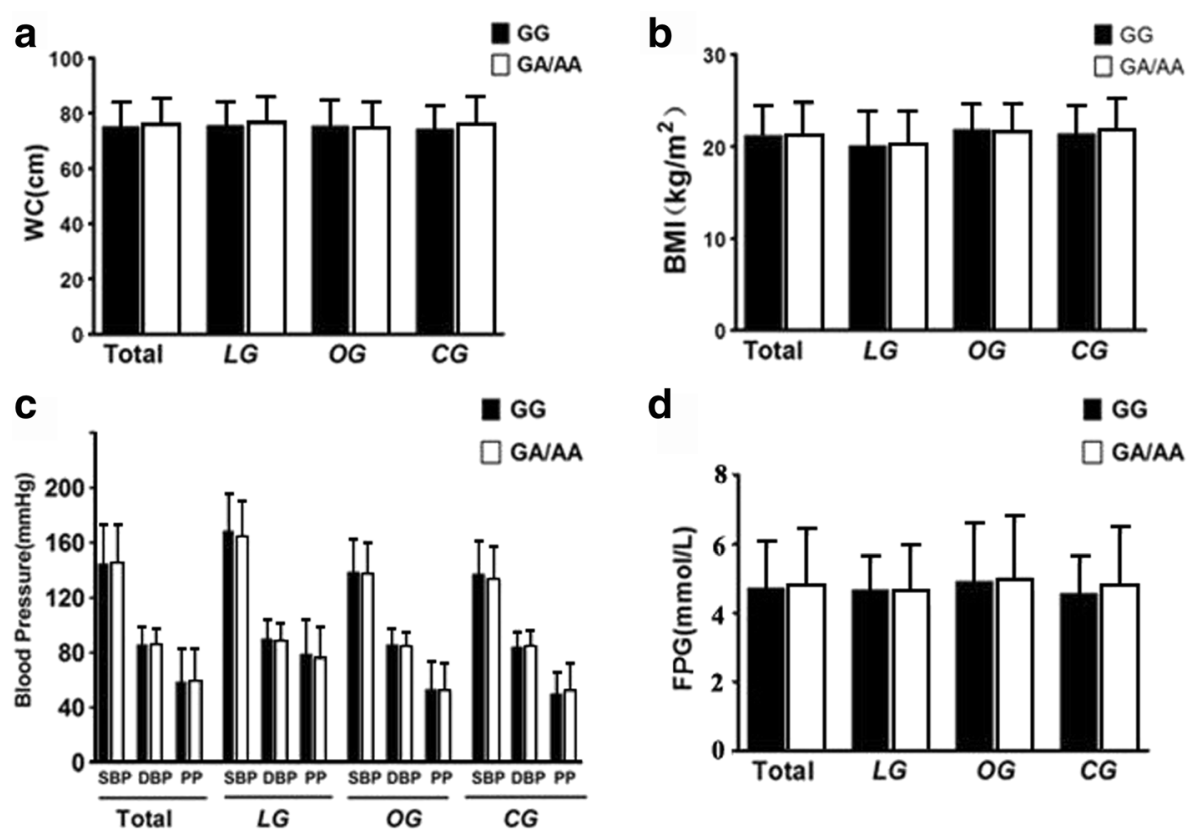

e

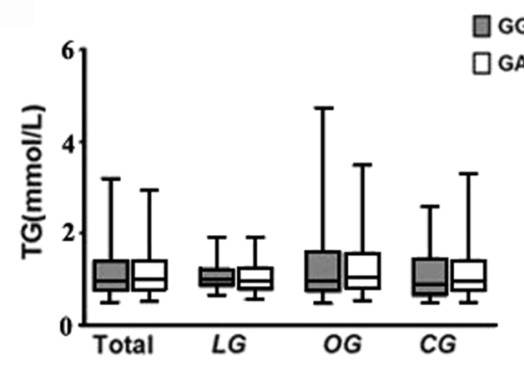

f

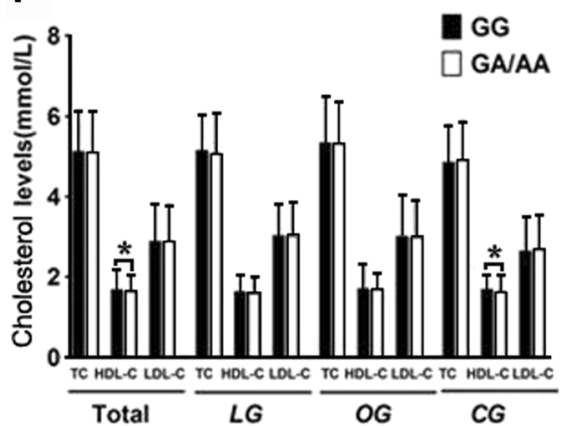

Fig. 2 Overall association of BDNF Val66Met genotypes with metabolic risk parameters. (a) with WC; (b) with BMI; (c) with blood pressure; (d) with FPG; (e) with TG; (f) with cholesterol levels. TG value was presented as Whiskers 5-95 percentile while the values of other parameters were presented as Mean $\pm S D$. * indicates $P<0.05$. Abbreviations: $L G$, long-lived group; OG, offspring group; CG, control group; WC, waist circumference; BMI, body mass index; FPG, fasting plasma glucose; SBP, systolic blood pressure; DBP, diastolic blood pressure; PP, pulse pressure; TC, total cholesterol; TG, triglyceride; HDL-C, high-density lipoprotein cholesterol; LDL-C, low-density lipoprotein cholesterol

Val66Met and obese phenotype which might be modulated by other factors such as ethnic background, age, gender, living environment, dietary structure and lifestyle [34]. Intriguingly, the Met allele carriers in OG exhibited a lower BMI level, implying that the descendants of nonagenarians may endow other unraveled buffering genotypes which may attenuate the unfavorable effect of this variant. In addition, the individuals with Met allele in the overweight subclass of CG presented a higher waist circumference, indicating that Met is mainly linked to abdominal obesity.

\section{BDNF and blood pressure}

Emerging evidences show that BDNF might be involved in the regulation of arterial pressure via different pathways. For instance, it has been recently demonstrated that long-term elevation of BDNF in the paraventricular nucleus of the hypothalamus has a major impact on central regulation of sympathetic activity, blood pressure and heart rate which is mediated by ANG II/angiotensin type 1 receptor and angiotensin/ Mas [35]. Dietary high salt intake promotes vasopressin release which contributes to the elevation of arterial pressure through BDNF-TrkB-GABAergic signaling system [35-37]. BDNF Val66Met modulates hypothalamicpituitary-adrenal axis reactivity and regulation, with women bearing $\mathrm{Val} / \mathrm{Met}$ genotype and men bearing Val/Val being particularly vulnerable to psychological stress [38], an essential etiology for primary hypertension. These data implicate that higher central or circulating BDNF may account for arterial hypertension 

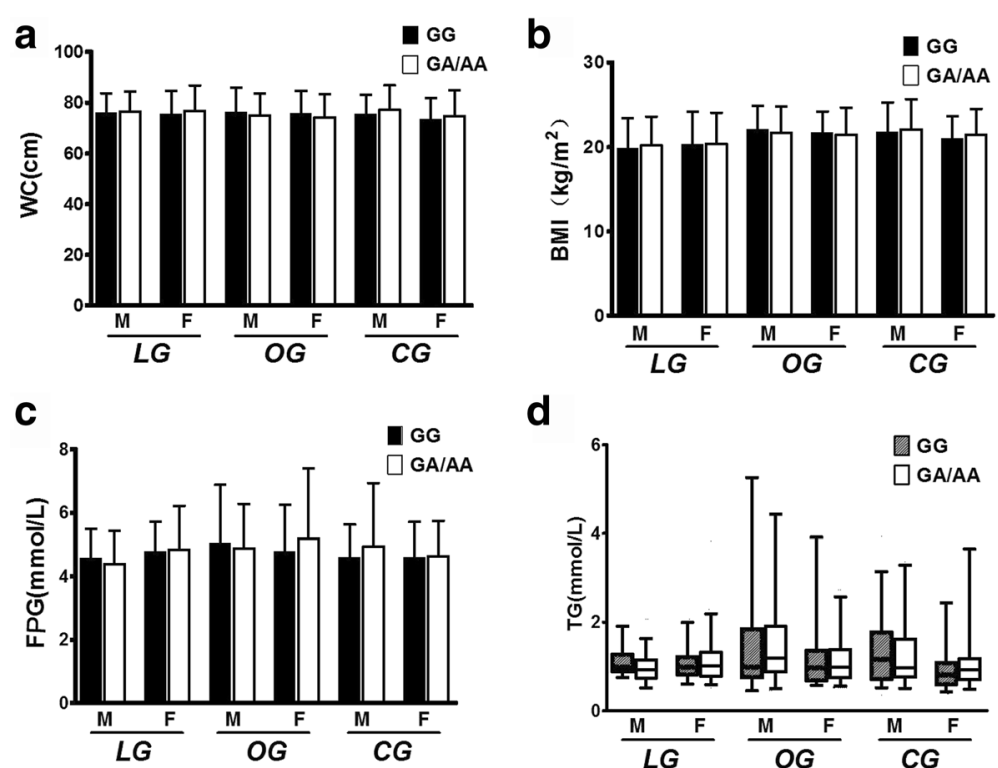

d
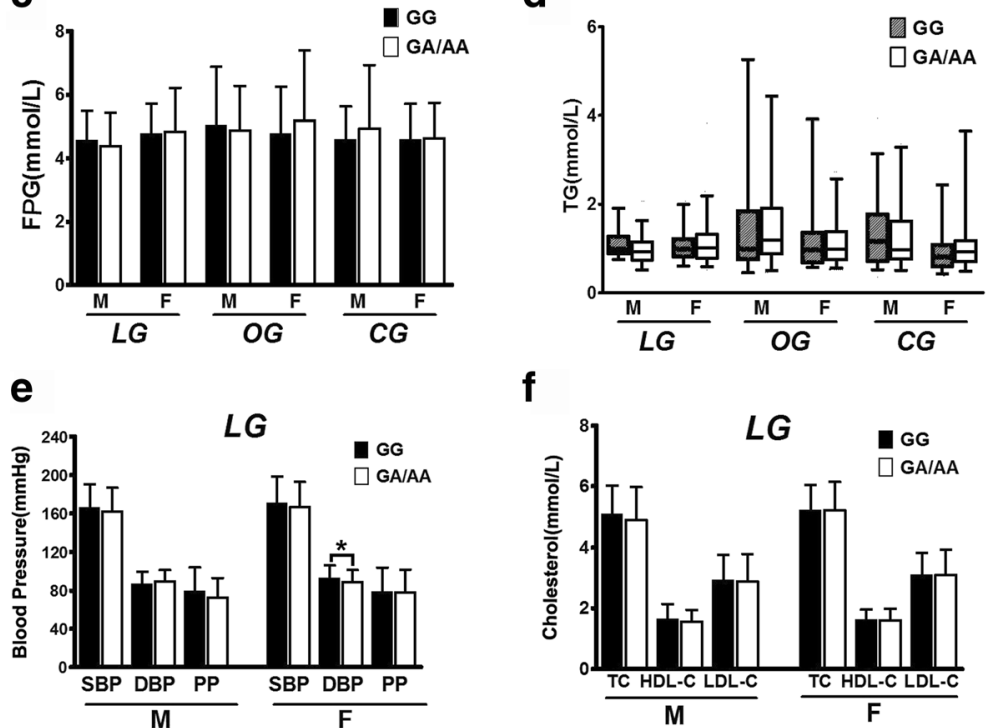

f
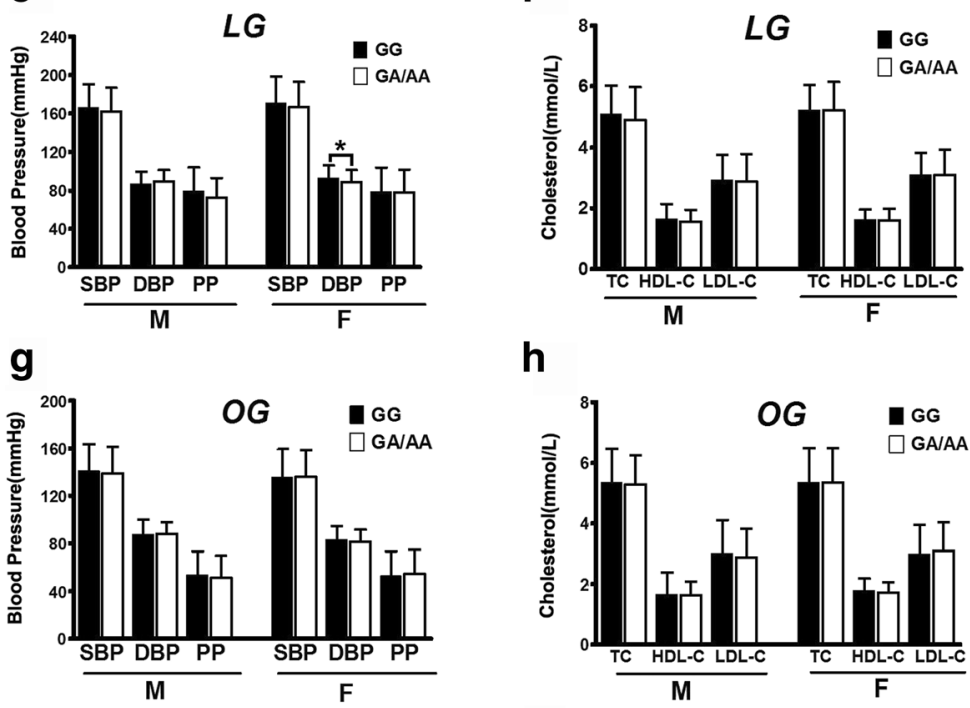

h
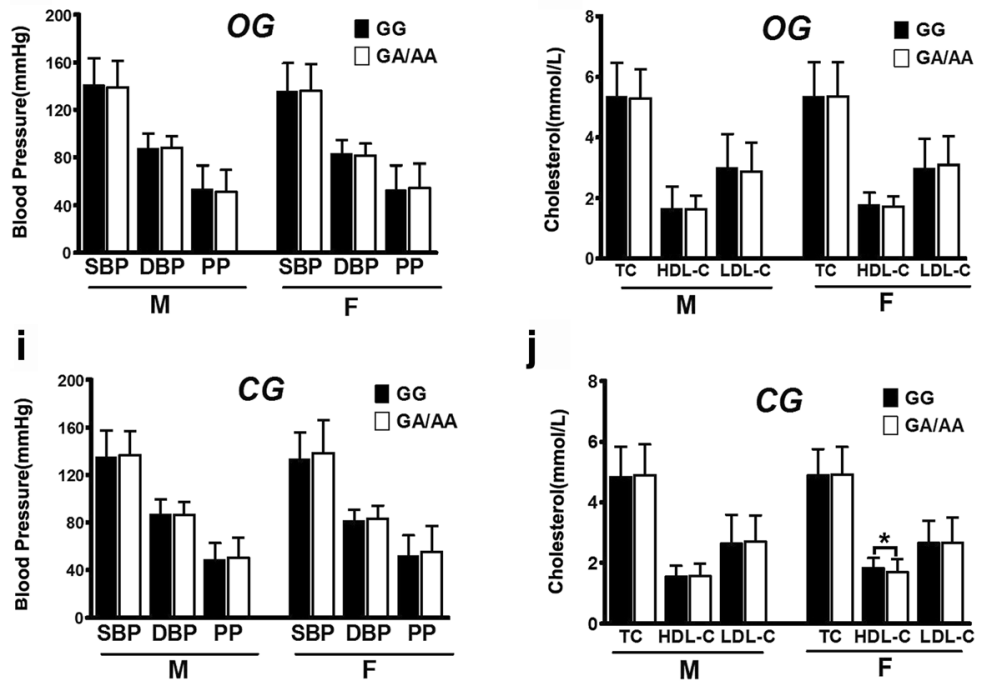

Fig. 3 Association of BDNF Val66Met genotypes with metabolic risk parameters stratified by sex. With WC (a), BMI (b), FPG (c) and TG (d) in the three groups investigated; with blood pressure and cholesterol levels in LG (e and $\mathbf{f}$, respectively), in OG (g and $\mathbf{h}$, respectively) and in OC (i and $\mathbf{j}$, respectively). Not all association panels is presented in this figure. M, males; F, females. Other notes and abbreviations see legends of Fig. 2

and Met variant may link to a reduced BDNF level and is favorable to better blood pressure regulation. However, we detected an elevated but not lowered SBP level in A genotype carriers in the normal BMI subgroups of the controls and an elevated DBP level in the hyperglycemic subgroup of OG when compared to non-A genotype carriers. We have no further explanation for this result due to the lack of serum BDNF level and other functional experiments on this polymorphism. 
Table 2 Correlation between parameters and the BDNF Val66Met polymorphism

\begin{tabular}{|c|c|c|c|c|c|c|}
\hline Parameter & Relative factor & Unstandardized coefficients & Standard error & Standardized coefficients & $t$ & $P$ \\
\hline \multicolumn{7}{|l|}{$\overline{L G}$} \\
\hline \multirow[t]{3}{*}{ BMI } & WC & 0.118 & 0.020 & 0.296 & 5.924 & 0.000 \\
\hline & $\mathrm{HC}$ & 0.184 & 0.025 & 0.342 & 7.333 & 0.000 \\
\hline & DBP & -0.498 & 0.227 & -0.072 & -2.193 & 0.029 \\
\hline \multirow[t]{3}{*}{ FBG } & Sex & 0.353 & 0.106 & 0.147 & 3.326 & 0.001 \\
\hline & $\mathrm{TG}$ & 0.391 & 0.122 & 0.143 & 3.217 & 0.001 \\
\hline & WC & 0.012 & 0.006 & 0.093 & 2.094 & 0.037 \\
\hline SBP & Sex & 4.815 & 2.401 & 0.091 & 2.005 & 0.045 \\
\hline \multirow[t]{2}{*}{ DBP } & $\mathrm{TC}$ & 1.879 & 0.586 & 0.143 & 3.208 & 0.001 \\
\hline & $\mathrm{BMl}$ & 0.484 & 0.162 & 0.133 & 2.995 & 0.003 \\
\hline PP & - & - & - & - & - & - \\
\hline \multirow[t]{4}{*}{ TC } & DBP & 0.011 & 0.003 & 0.139 & 3.146 & 0.002 \\
\hline & Sex & 0.223 & 0.091 & 0.110 & 2.445 & 0.015 \\
\hline & FBG & 0.088 & 0.038 & 0.103 & 2.306 & 0.022 \\
\hline & Genotype & -0.132 & 0.063 & -0.093 & -2.090 & 0.037 \\
\hline \multirow[t]{2}{*}{ TG } & FBG & 0.058 & 0.016 & 0.159 & 3.556 & 0.000 \\
\hline & DBP & 0.004 & 0.001 & 0.108 & 2.425 & 0.016 \\
\hline $\mathrm{HDL}-\mathrm{C}$ & $\mathrm{BMI}$ & -0.015 & 0.007 & -0.105 & -2.336 & 0.020 \\
\hline \multirow[t]{2}{*}{ LDL-C } & DBP & 0.009 & 0.003 & 0.131 & 2.930 & 0.004 \\
\hline & Sex & 0.230 & 0.079 & 0.131 & 2.921 & 0.004 \\
\hline \multicolumn{7}{|l|}{ OG } \\
\hline \multirow[t]{4}{*}{ BMI } & WC & 0.090 & 0.037 & 0.054 & 2.427 & 0.016 \\
\hline & Sex & 0.730 & 0.144 & 0.121 & 5.062 & 0.000 \\
\hline & DBP & 0.040 & 0.006 & 0.148 & 6.502 & 0.000 \\
\hline & Age & 0.171 & 0.067 & 0.059 & 2.554 & 0.011 \\
\hline \multirow[t]{2}{*}{ FBG } & WC & -0.041 & 0.011 & -0.173 & -3.691 & 0.000 \\
\hline & DBP & -0.649 & 0.131 & -0.205 & -4.952 & 0.000 \\
\hline \multirow[t]{3}{*}{ SBP } & Age & 0.902 & 0.096 & 0.356 & 9.377 & 0.000 \\
\hline & $\mathrm{BMI}$ & 1.522 & 0.290 & 0.199 & 5.251 & 0.000 \\
\hline & Sex & -4.215 & 1.751 & -0.091 & -2.407 & 0.016 \\
\hline \multirow[t]{4}{*}{ DBP } & $\mathrm{BMI}$ & 1.669 & 0.172 & 0.447 & 9.717 & 0.000 \\
\hline & Sex & -4.944 & 0.838 & -0.219 & -5.897 & 0.000 \\
\hline & $\mathrm{HC}$ & -0.924 & 0.421 & -0.085 & -2.197 & 0.028 \\
\hline & FBG & -0.651 & 0.235 & -0.105 & -2.767 & 0.006 \\
\hline PP & Age & 0.859 & 0.083 & 0.393 & 10.389 & 0.000 \\
\hline \multirow[t]{4}{*}{ TC } & WC & -0.037 & 0.013 & -0.213 & -2.911 & 0.004 \\
\hline & Age & -0.013 & 0.005 & -0.105 & -2.670 & 0.008 \\
\hline & BMI & 0.069 & 0.027 & 0.182 & 2.588 & 0.010 \\
\hline & DBP & -0.008 & 0.004 & -0.084 & -2.065 & 0.039 \\
\hline \multirow[t]{2}{*}{ TG } & WC & 0.057 & 0.011 & 0.353 & 5.282 & 0.000 \\
\hline & Sex & -0.076 & 0.033 & -0.151 & -2.287 & 0.023 \\
\hline \multirow[t]{2}{*}{ HDL-C } & WC & -0.012 & 0.002 & -0.189 & -4.676 & 0.000 \\
\hline & Sex & 0.115 & 0.045 & 0.100 & 2.543 & 0.011 \\
\hline LDL-C & $\mathrm{BMI}$ & 0.113 & 0.014 & 0.328 & 7.871 & 0.000 \\
\hline
\end{tabular}


Table 2 Correlation between parameters and the BDNF Val66Met polymorphism (Continued)

\begin{tabular}{|c|c|c|c|c|c|c|}
\hline & DBP & -0.012 & 0.004 & -0.125 & -2.996 & 0.003 \\
\hline \multicolumn{7}{|l|}{$C G$} \\
\hline \multirow[t]{2}{*}{ BMI } & WC & 0.151 & 0.025 & 0.278 & 5.998 & 0.000 \\
\hline & Age & 0.247 & 0.079 & 0.084 & 3.123 & 0.002 \\
\hline FBG & WC & 0.186 & 0.055 & 0.137 & 3.359 & 0.001 \\
\hline \multirow[t]{2}{*}{ SBP } & Age & -4.021 & 1.801 & -0.086 & -2.232 & 0.026 \\
\hline & $\mathrm{HC}$ & 0.706 & 0.152 & 0.181 & 4.631 & 0.000 \\
\hline \multirow[t]{2}{*}{ DBP } & Sex & 0.541 & 0.142 & 0.157 & 3.822 & 0.000 \\
\hline & FBG & -0.650 & 0.305 & -0.087 & -2.133 & 0.033 \\
\hline \multirow[t]{3}{*}{ PP } & Age & -3.783 & 1.391 & -0.104 & -2.720 & 0.007 \\
\hline & $\mathrm{HC}$ & 0.429 & 0.114 & 0.141 & 3.751 & 0.000 \\
\hline & Sex & 4.185 & 1.404 & 0.113 & 2.980 & 0.003 \\
\hline \multirow[t]{4}{*}{ TC } & BMI & 0.045 & 0.020 & 0.149 & 2.310 & 0.021 \\
\hline & Age & -0.015 & 0.004 & -0.143 & -3.320 & 0.001 \\
\hline & CC & -0.040 & 0.011 & -0.275 & -3.737 & 0.000 \\
\hline & WC & 0.026 & 0.008 & 0.243 & 3.117 & 0.002 \\
\hline \multirow[t]{5}{*}{ TG } & $\mathrm{BMI}$ & 0.076 & 0.021 & 0.223 & 3.586 & 0.000 \\
\hline & Age & -0.027 & 0.005 & -0.233 & -5.740 & 0.000 \\
\hline & FBG & 0.096 & 0.029 & 0.131 & 3.270 & 0.001 \\
\hline & $\mathrm{HC}$ & -0.058 & 0.013 & -0.315 & -4.616 & 0.000 \\
\hline & WC & 0.029 & 0.009 & 0.246 & 3.365 & 0.001 \\
\hline \multirow[t]{2}{*}{ HDL-C } & Sex & -0.003 & 0.001 & -0.108 & -2.688 & 0.007 \\
\hline & WC & -0.008 & 0.002 & -0.154 & -3.827 & 0.000 \\
\hline LDL-C & WC & 0.012 & 0.004 & 0.120 & 2.914 & 0.004 \\
\hline
\end{tabular}

Abbreviations: $L G$ long-lived group, OG offspring group, CG control group, WC waist circumference, $H C$ hip circumference, $C C$ chest circumference, $B M I$ body mass index, FPG fasting plasma glucose, SBP systolic blood pressure, DBP diastolic blood pressure, $P P$ pulse pressure, $T C$ total cholesterol, $T G$ triglyceride, $H D L-C$ high-density lipoprotein cholesterol, LDL-C low-density lipoprotein cholesterol

\section{BDNF Val66Met and glucose metabolism}

As mentioned above, previous studies on model animals had demonstrated that BDNF may suppress food uptake, improve insulin resistance and maintain glucose homeostasis [28, 29]. Intracerebroventricular or intraperitoneal administration of BDNF results in a reduction of plasma glucose level of diabetic or insulin deficient mice [10]. Clinical epidemiological investigation also observed that the serum BDNF level of diabetic patients were significantly lower than that of normal controls and were reversely correlated with blood glucose concentration independent of obesity, indicating the robust relationship of BDNF and glucose metabolism in that the hyperglycemic status of diabetic patients might have repressed the release of BDNF from the brain [39, 40]. Furthermore, male BDNF Met/Met carriers with clozapine-induced metabolic syndrome exhibited significant higher fasting glucose levels than those with $\mathrm{Val} / \mathrm{Val}$ or $\mathrm{Val} / \mathrm{Met}$ genotypes [14]; carriers of Met allele displayed elevated blood glucose and predicted lower memory scores [41]. These observations demonstrated a pronounced influence of
BDNF on glucose homeostasis. However, Bonaccorso et al. [13] detected no different glucose level between Met and non-Met carriers in patients with bipolar and schizoaffective disorder, indicating the uncertainty affecting pattern of BDNF on glucose metabolism. In the present study, a lowered FPG was only noted in the BDFN GA/ AA carriers of euglycemic and normolipidemic subgroup of LG but not in any genotype of other groups, implying a limited effect of BDNF rs6265 on blood glucose modulation in our studying populations. The relatively lower average FPG levels and lower hyperglycemia prevalence in these populations might be one of the explanations for this observation.

\section{BDNF Val66Met and lipid metabolism}

Information regarding the influence of BDNF on lipid modulation is scarce. Tsuchida and colleagues were some of the researchers who firstly focused on the effect of BDNF on lipid metabolism and noted that subcutaneous injection of BDNF (twice/week) could significantly improve lipid and glucose profiles in diabetic mice [42]. 
In humans, serum BDNF levels were found to be significantly higher in type 2 diabetic patients than in the healthy controls and positively correlated with triglyceride level [43]. As compared to non-Met, BDNF 66Met carriers with bipolar disorder showed a greater increase in triglycerides and triglycerides/HDL-C ratio after three or six months of antipsychotic therapy [13]. We noted herein similar correlation trend between BDNF Val66Met and lipid profiles where Met allele was essentially linked to elevated triglyceride but lowered HDL-C level relative to non-Met bearers, particularly in the control and offspring groups. Tsuchida et al. postulated that BDNF might enter the brain and act on the hypothalamic neurons, leading to activation of sympathetic nerve system, enhancement of energy expenditure and reduction of lipid accumulation as does leptin [42]. It is thus reasonable to assume that the derived Met allele may account for a lower BDNF level and a reduced energy consumption and lipid increment in our participants.

\section{BDNF Val66Met and neuroinflammation}

Normal aging has been regarded as a chronic and lowgrade pro-inflammatory state, with an over-expression of systemic inflammatory cytokines including IL-1, IL-6 and TNF- $\alpha$ etc. A number of highly prevalent risk factors such as obesity, diabetes, hypertension, and atherosclerosis are increasingly believed to silently contribute to systemic- and neuro-inflammation [44-46]. Cerebral small vessel disease and perfusion deficits resulting from these pathological conditions may account for the neuroinflammation and subsequent neurodegeneration and cognitive dysfunction [46]. BDNF has been implicated to play a neuroprotective role in the central nerve system; low levels of BDNF have been associated with aging and an array of neurological and psychiatric disorders $[47,48]$. One of the explanations for this protection in that it counteracts the effects of some inflammatory cytokines such as C-reactive protein [49]. It is thus reasonable to assume that any BDNF polymorphism with lower BDNF activity may link to enhanced neuroinflammation and worsened cognitive outcome. This notion is underscored by the observation of Dooley and colleagues who noted a positive association between $\mathrm{C}$-reactive protein levels and cognitive depressive symptoms among BDNF Met carriers of breast cancer survivors [50]. Therefore, we postulate that the control females who were enriched with BDNF Met allele in our study may also have higher inflammatory cytokines and poorer cognition, however this needs to be confirmed in our future longitudinal follow-up.

\section{Limitations}

The first limitation of the present study is that we only focused on BDNF genotype, without assessing the circulating
BDNF protein levels. There are several intervening aspects between an individual's genotype and its function, therefore an individual's BDNF genotype does not necessarily determine BDNF protein levels. Although previous study has shown a correlation between the Val66Met polymorphism and circulating BDNF level [51], indicating that BDNF genotype may serve as a proxy for protein levels at least in some samples, others have not found this association [52]. The second limitation is that we failed to detect inflammatory biomarkers (e.g. CRP, IL-1, 6, TNF- $\alpha$ ) which are very important parameters in evaluating the association of BDNF genotype with neuroinflammaging. Finally, we failed to collect data on cognitive status (e.g. MMSE score) and life style (e.g. dietary habit, alcohol consumption, smoking and physical activity) which may be potential confounders on our results. All these parameters and data will be considered in our future research on these populations.

\section{Conclusions}

In conclusion, the key finding of the current work is that BDNF Met variant represents more frequently and correlates with elevated BMI and SBP levels and lowered HDL-C level in the average population inhabiting the river basin of Hongshuihe, which may be some of the contributors to their poorer survivorship as compared to whom achieve prolonged longevity. Further investigations on the interactions among serum BDNF and insulin levels and metabolic measures are warranted.

\section{Additional files}

Additional file 1: Figure S1. Association between BDNF Val66Met genotypes and metabolic risk parameters stratified by BMI status, N, normal; OW, overweight; other notes and abbreviations see legends of Fig. 3. (JPG $1311 \mathrm{~kb})$

Additional file 2: Figure S2. Association between BDNF Val66Met genotypes and metabolic risk parameters stratified by FPG status, E, euglycaemia; $H$, hyperglyceamia. Other notes and abbreviations see legends of Fig. 3. (JPG $1271 \mathrm{~kb}$ )

Additional file 3: Figure S3. Association between BDNF Val66Met genotypes and metabolic risk parameters stratified by lipid status, N, normolipidemia; $H$, hyperlipidemia. Other notes and abbreviations see legends of Fig. 3. (JPG 1289 kb)

\section{Abbreviations \\ AD: Alzheimer's disease; BDNF: Brain-derived neurotrophic factor; BMI: Body mass index; Cl: Confidential interval; CNS: Central nervous system; DBP: Diastolic blood pressure; FPG: Fasting plasma glucose; HDL-C: High density lipoprotein cholesterol; LDL-C: Low density lipoprotein cholesterol; OR: Odds ratio; PD: Parkinson's disease; SBP: Systolic blood pressure; TC: Total cholesterol; TG: Triglycerides}

\section{Acknowledgments}

We thank all participants for their sample donation. We specifically appreciate Dr. Jian-Hua Huang (Fengshan People's Hospital), Dr. Hong-Bo Liang (Donglan People's Hospital), Dr. Jin-Zhu Huang (Du'an People's Hospital) and Dr. Wen Shi (Pingguo Bureau of Health) for the organization of sampling and field studies. 


\section{Funding}

This work was supported by National Natural Science Foundation of China (No. 31160209, 81360066) and the Natural Science Foundation of Guangxi (2013GXNSFAA019180, 2013GXNSFBB053002). The funders had no role in study design, data collection and analysis, decision to publish, or preparation of the manuscript.

\section{Availability of data and materials}

Data used in this study will be made available for non-commercial research and validation purposes, upon request.

\section{Authors' contributions}

Conceived and designed the experiments: J-HP and S-LP. Performed the experiments: J-HP, C-WL, H-YW, Q-HL, R-JG and LH. Analyzed the data: J-HP. Contributed reagents/materials/analysis tools: J-HP. and S-LP. Wrote the paper: J-HP. and S-LP. Carried out the epidemiological survey and collected the samples: J-HP, C-WL, S-LP, YD, Z-YB, HH, Z-PL, X-LZ and R-XY. All authors read and approved the final manuscript.

\section{Competing interests}

The authors declare that they have no competing interests.

\section{Consent for publication}

Not applicable.

\section{Ethics approval and consent to participate}

The current study was approved by the Ethics Committee of Guangxi Medical University. All participants were given verbal informed consents and their fingerprints (to express consent) were obtained after they received a full explanation of the study. Written informed consents were not obtained because of their low educational level. The consent procedure was also approved by the Ethics Committee of Guangxi Medical University.

\section{Author details}

${ }^{1}$ Department of Pathophysiology, Guangxi Medical University, 22 Shuangyong Road, Nanning 530021, Guangxi, China. ${ }^{2}$ Guangxi Colleges and Universities Key Laboratory of Human Development and Disease Research, 22 Shuangyong Road, Nanning 530021, Guangxi, China. ${ }^{3}$ Department of Neurology, Jiangbin Hospital of Guangxi Zhuang Autonomous Region, 85 Hedi Road, Nanning 530021, Guangxi, China. ${ }^{4}$ Department of Cardiology, The First Affiliated Hospital of Guangxi Medical University, 22 Shuangyong Road, Nanning 530021, Guangxi, China.

Received: 8 July 2016 Accepted: 8 December 2016 Published online: 05 January 2017

\section{References}

1. Binder DK, Scharfman HE. Brain-derived neurotrophic factor. Growth Factors. 2004;22:123-31.

2. Murer MG, Yan Q, Raisman-Vozari R. Brain-derived neurotrophic factor in the control human brain, and in Alzheimer's disease and Parkinson's disease. Prog Neurobiol. 2001;63:71-124.

3. Shimada $H$, Makizako $H$, Doi T, Yoshida D, Tsutsumimoto $K$, Anan $Y$, et al. A large, cross-sectional observational study of serum BDNF, cognitive function, and mild cognitive impairment in the elderly. Front Aging Neurosci. 2014;6:69

4. Pan W, Banks WA, Fasold MB, Bluth J, Kastin AJ. Transport of brain-derived neurotrophic factor across the bloodbrain barrier. Neuropharmacology. 1998:37:1553-61.

5. Rasmussen P, Brassard P, Adser H, Pedersen MV, Leick L, Hart E, et al. Evidence for a release of brain-derived neurotrophic factor from the brain during exercise. Exp Physiol. 2009;94:1062-9.

6. Zoladz JA, Pilc A, Majerczak J, Grandys M, Zapart-Bukowska J, Duda K Endurance training increases plasma brain-derived neurotrophic facto concentration in young healthy men. J Physiol Pharmacol. 2008:59 Suppl 7: $119-32$.

7. Petryshen TL, Sabeti PC, Aldinger KA, Fry B, Fan JB, Schaffner SF, et al. Population genetic study of the brain-derived neurotrophic factor (BDNF) gene. Mol Psychiatry. 2010;15:810-5.

8. Chaldakov G. The metabotrophic NGF and BDNF: an emerging concept. Arch Ital Biol. 2011;149:257-63.
9. Soppet D, Escandon E, Maragos J, Middlemas DS, Reid SW, Blair J, et al. The neurotrophic factors brain-derived neurotrophic factor and neurotrophin-3 are ligands for the trkB tyrosine kinase receptor. Cell. 1991;65:895-903.

10. Nakagawa T, Tsuchida A, Itakura Y, Nonomura T, Ono M, Hirota F, et al. Brain-derived neurotrophic factor regulates glucose metabolism by modulating energy balance in diabetic mice. Diabetes. 2000;49:436-44.

11. Hanson IM, Seawright A, van Heyningen V. The human BDNF gene maps between FSHB and HVBS1 at the boundary of 11p13-p14. Genomics. 1992; 13:1331-3.

12. Egan MF, Kojima M, Callicott JH, Goldberg TE, Kolachana BS, Bertolino A, et al. The BDNF val66met polymorphism affects activity-dependent seretion of BDNF and human memory and hippocampal function. Cell. 2003;112: 257-69.

13. Bonaccorso S, Sodhi M, Li J, Bobo WV, Chen Y, Tumuklu M, et al. The brainderived neurotrophic factor (BDNF) Val66Met polymorphism is associated with increased body mass index and insulin resistance measures in bipolar disorder and schizophrenia. Bipolar Disord. 2015:17:528-35.

14. Zhang Y, Chen M, Wu Z, Chen J, Yu S, Fang Y, et al. Association study of Val66Met polymorphism in brain-derived neurotrophic factor gene with clozapine-induced metabolic syndrome: preliminary results. PLoS ONE. 2013; 8:e72652.

15. Zhao X, Xi B, Shen Y, Wu L, Hou D, Cheng H, et al. An obesity genetic risk score is associated with metabolic syndrome in Chinese children. Gene. 2014;535:299-302.

16. Lv ZP, Zheng CG, Yang Z, Liang JY, Hu CY, Li X, et al. Cognitive function and activity of daily life of the elderly of Zhuang nationality in Bama County. Chin Mental Health J. 2003;17:98-100.

17. Luo CY, Liu CW, Ge L, Pang GF, Yang M, Hu CY, et al. PPARD +294C overrepresentation in general and long-lived population in China Bama longevity area and unique relationships between PPARD +294 T/C polymorphism and serum lipid profiles. Lipids Health Dis. 2015;14:17.

18. Peng J, Sun P, Liu C, Pan S. Correlation Between the BDNF Val66Met Polymorphisms and Metabolic Risks in Long-Lived Zhuang Population. J Am Coll Cardiol. 2015;66:Suppl S. C35.

19. Li Q, Yin RX, Wei XL, Yan TT, Aung LH, Wu DF, et al. ATP-binding cassette transporter G5 and G8 polymorphisms and several environmental factors with serum lipid levels. PLOS ONE. 2012;7:e37972.

20. Pan SL, Wang F, Lu ZP, Liu CW, Hu CY, Luo H, et al. Cholesteryl ester transfer protein TaqIB polymorphism and its association with serum lipid levels and longevity in Chinese Bama Zhuang population. Lipids Health Dis. 2012;11:26.

21. Ruixing $Y$, Yuming $C$, Shangling $P$, Fengping $H$, Tangwei $L$, Dezhai $Y$, et al. Effects of demographic, dietary and other lifestyle factors on the prevalence of hyperlipidemia in Guangxi Hei Yi Zhuang and Han populations. Eur J Cardiovasc Prev Rehabil. 2006;13:977-84.

22. Sheikh HI, Hayden EP, Kryski KR, Smith HJ, Singh SM. Genotyping the BDNF rs6265 (val66met) polymorphism by one-step amplified refractory mutation system PCR. Psychiatr Genet. 2010;20:109-12.

23. Xu B, Goulding EH, Zang K, Cepoi D, Cone RD, Jones KR, et al. Brain-derived neurotrophic factor regulates energy balance downstream of melanocortin4 receptor. Nat Neurosci. 2003;6:736-42.

24. Unger TJ, Calderon GA, Bradley LC, Sena-Esteves M, Rios M. Selective deletion of Bdnf in the ventromedial and dorsomedial hypothalamus of adult mice results in hyperphagic behavior and obesity. J Neurosci. 2007;27: 14265-74.

25. Akkermann K, Hiio K, Villa I, Harro J. Food restriction leads to binge eating dependent upon the effect of the brain-derived neurotrophic factor Val66Met polymorphism. Psychiatry Res. 2011;185:39-43.

26. Skledar M, Nikolac M, Dodig-Curkovic K, Curkovic M, Borovecki F, Pivac N. Association between brain-derived neurotrophic factor Val66Met and obesity in children and adolescents. Prog Neuropsychopharmacol Biol Psychiatry. 2012;36:136-40.

27. Ma XY, Qiu WQ, Smith CE, Parnell LD, Jiang ZY, Ordovas JM, et al. Association betweenBDNF rs6265 and Obesity in the Boston Puerto Rican Health Study. J Obes. 2012;2012:102942.

28. Ono $M$, Ichihara J, Nonomura $T$, Itakura $Y$, Taiji $M$, Nakayama $C$, et al. Brain-derived neurotrophic factor reduces bloodglucose level in obese diabetic mice but not in normal mice. Biochem Biophys Res Commun. 1997;238:633-7.

29. Duan W, Guo Z, Jiang H, Ware M, Mattson MP. Reversal of behavioral and metabolic abnormalities, and insulin resistance syndrome, by dietary 
restriction in mice deficient in brain-derived neurotrophic factor. Endocrinology. 2003;144:2446-53.

30. Shugart YY, Chen L, Day IN, Lewis SJ, Timpson NJ, et al. Two British women studies replicated the association between the Val66Met polymorphism in the brain-derivedneurotrophic factor (BDNF) and BMI. Eur J Hum Genet. 2009;17:1050-5.

31. Wu L, Xi B, Zhang M, Shen $Y$, Zhao X, Cheng $H$, et al. Associations of six single nucleotide polymorphisms in obesity-related genes with $\mathrm{BMI}$ and risk of obesity in Chinese children. Diabetes. 2010;59:3085-9.

32. Hong KW, Lim JE, Go MJ, Shin Cho Y, Ahn Y, Han BG, et al. Recapitulation of the association of the Val66Met polymorphism of BDNF gene with BMI in Koreans. Obesity (Silver Spring). 2012;20:1871-5.

33. Friedel S, Horro FF, Wermter AK, Geller F, Dempfle A, Reichwald K, et al. Mutation screen of the brain derived neurotrophic factor gene (BDNF): identification of several genetic variants and association studies in patients with obesity, eating disorders, and attentiondeficit/hyperactivity disorder. Am J Med Genet B Neuropsychiatr Genet. 2005;132B:96-9.

34. Erdos B, Backes I, McCowan ML, Hayward LF, Scheuer DA. Brain-derived neurotrophic factor modulates angiotensin signaling in the hypothalamus to increase blood pressure in rats. Am J Physiol Heart Circ Physiol. 2015;308: H612-22.

35. Choe KY, Han SY, Gaub P, Shell B, Voisin DL, Knapp BA, et al. High salt intake increases blood pressure via BDNF-mediated downregulation of KCC2 and impaired baroreflex inhibition of vasopressin neurons. Neuron. 2015;85:549-60.

36. Amoureux S, Lorgis L, Sicard P, Girard C, Rochette L, Vergely C. Vascular BDNF expression and oxidative stress during aging and the development of chronic hypertension. Fundam Clin Pharmacol. 2012;26:227-34.

37. Chan SH, Wu CW, Chang AY, Hsu KS, Chan JY. Transcriptional Upregulation of Brain-Derived Neurotrophic Factor in Rostral Ventrolateral Medulla by Angiotensin II. Circ Res. 2010;107:1127-39.

38. Shalev I, Lerer E, Israel S, Uzefovsky F, Gritsenko I, Mankuta D, et al. BDNF Val66Met polymorphism is associated with HPA axis reactivity to psychological stress characterized by genotype and gender interactions. Psychoneuroendocrinology. 2009;34:382-8.

39. Krabbe KS, Nielsen AR, Krogh-Madsen R, Plomgaard P, Rasmussen $P$, Erikstrup C, et al. Brain-derived neurotrophic factor (BDNF) and type 2 diabetes. Diabetologia. 2007;50:431-8.

40. Fujinami A, Ohta K, Obayashi H, Fukui M, Hasegawa G, Nakamura N, et al. Serum Brain-derived neurotrophic factor in patients with type 2 diabetes mellitus: Relationship to glucose metabolism and biomarkers of insulin resistance. Clin Biochem. 2008;41:812-7.

41. Raz N, Dahle CL, Rodrigue KM, Kennedy KM, Land SJ, Jacobs BS. Brainderived neurotrophic factor Val66Met and blood glucose: a synergistic effect on memory. Front Hum Neurosci. 2008;2:12.

42. Tsuchida A, Nonomura T, Nakagawa T, Itakura Y, Ono-Kishino M, Yamanaka $\mathrm{M}$, et al. Brain-derived neurotrophic factor ameliorates lipid metabolism in diabetic mice. Diabetes Obes Metab. 2002;4:262-9.

43. Boyuk B, Degirmencioglu S, Atalay H, Guzel S, Acar A, Celebi A, et al. Relationship between levels of brain-derived neurotrophic factor and metabolic parameters in patients with type 2 diabetes mellitus. J Diabetes Res. 2014;2014:978143.

44. Bruunsgaard H, Pedersen M, Pedersen BK. Aging and proinflammatory cytokines. Curr Opin Hematol. 2001;8:131-6.

45. Sandu RE, Buga AM, Uzoni A, Petcu EB, Popa-Wagner A. Neuroinflammation and comorbidities are frequently ignored factors in CNS pathology. Neural Regen Res. 2015;10:1349-55.

46. Popa-Wagner A, Buga AM, Tica AA, Albu CV. Perfusion deficits, inflammation and aging precipitate depressive behaviour. Biogerontology. 2014;15:439-48.

47. Smith AJ, Malan L, Uys AS, Malan NT, Harvey BH, Ziemssen T. Attenuated brain-derived neurotrophic factor and hypertrophic remodelling: the SABPA study. J Hum Hypertens. 2015;29:33-9.

48. Krabbe KS, Mortensen EL, Avlund K, Pedersen AN, Pedersen BK, Jørgensen T, et al. Brain-Derived Neurotrophic Factor Predicts Mortality Risk in Older Women. J Am Geriatr Soc. 2009;57:1447-52.

49. Noren Hooten N, Ejiogu N, Zonderman AB, Evans MK. Protective Effects of BDNF against C-Reactive Protein-Induced Inflammation in Women. Mediators Inflamm. 2015;2015:516783.
50. Dooley LN, Ganz PA, Cole SW, Crespi CM, Bower JE. Val66Met BDNF polymorphism as a vulnerability factor for inflammation-associated depressive symptoms in women with breast cancer. J Affect Disord. 2016; 197:43-50.

51. Lotrich FE, Albusaysi S, Ferrell RE. Brain-derived neurotrophic factor serum levels and genotype: association with depression during interferon-alpha treatment. Neuropsychopharmacology. 2013;38:985-95.

52. Terracciano A, Martin B, Ansari D, Tanaka T, Ferrucci L, Maudsley S, et al. Genes Brain Behav. 2010;9:512-8.

\section{Submit your next manuscript to BioMed Central and we will help you at every step:}

- We accept pre-submission inquiries

- Our selector tool helps you to find the most relevant journal

- We provide round the clock customer support

- Convenient online submission

- Thorough peer review

- Inclusion in PubMed and all major indexing services

- Maximum visibility for your research

Submit your manuscript at www.biomedcentral.com/submit 